\title{
Condições de trabalho de enfermagem e adoecimento: motivos para a redução da jornada de trabalho para 30 horas
}

Recebido em: 06/06/2012

Aceito em: 27/09/2012

Vanda Elisa Andres Felli ${ }^{1}$

O texto tem por objetivo fazer uma análise reflexiva sobre as condições de trabalho e adoecimento dos trabalhadores de enfermagem, determinadas por jornadas superiores a 30 horas. Partindo da análise do contexto do trabalho de enfermagem, o texto analisa as condições de trabalho e as suas implicações no adoecimento dos trabalhadores. Traz como consequências a diminuição da capacidade para o trabalho, o absenteísmo, a elevação de custos dos afastamentos, o impacto sobre o perfil de morbi-mortalidade dos trabalhadores e as implicações sobre a qualidade da assistência. Conclui-se que é necessário controlar a insalubridade, a periculosidade e a penosidade desse trabalho.

Descritores: Enfermagem, Saúde do Trabalhador, Condições de Trabalho, Jornada de Trabalho, Morbidade.

\section{Nursing working condition and sickness: reasons why to reduce the work journey to $\mathbf{3 0}$ hours}

The text aims to do a reflexive analysis about the working conditions and sickness of nursing workers, determined for journeys exceeding 30 hours. Based on the analysis of the context of nursing work, the text examines the working conditions and their implications in illness among workers. Also, the text brings as consequences the decreased capacity to work, absenteeism, higher costs of absence from work, the impact on morbidity and mortality profile of workers and the implications on the quality of care. It is concluded that it is necessary to control the insalubrity, dangerousness and painfulness of this work.

Descriptors: Nursing, Occupational Health, Working Conditions, Work Hours, Morbidity.

\section{Condiciones de trabajo de enfermería y enfermedad: motivos para la reducción de jornada para trabajar $\mathbf{3 0}$ horas}

El texto tiene como objetivo analizar reflexivamente sobre las condiciones de trabajo y enfermedades de los trabajadores de enfermería, determinado por turnos que excedan 30 horas. Con base en el análisis del contexto del trabajo de enfermería, el texto analiza las condiciones de trabajo y sus implicaciones en la enfermedad entre los trabajadores. Trae consecuencias como la disminución de la capacidad de trabajo, ausentismo, costos más altos de las ausencias, el impacto en lo perfil de morbilidad y mortalidad de los trabajadores y las implicaciones en la calidad de la atención. Se concluye que es necesario controlar la insalubridad, peligrosidad y doloroso trabajo.

Descriptores: Enfermería, Salud del Trabajador, Condiciones de Trabajo, Día de Trabajo, Morbidad.

\section{INTRODUÇÃO}

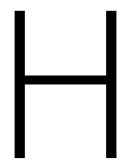

á a constatação de que a enfermagem, enquanto trabalho inserido no setor terciário da economia, na prestação de serviços de assistência à saúde, sofre o impacto das políticas sociais e econômicas capitalistas do País. Um dos impactos é constatado pelas más condições de trabalho, o que é inquestionável e comprovado pelo adoecimento dos trabalhadores, cujo adoecimento não tem transparência ou visibilidade nas estatísticas oficiais.

Para melhor compreensão do adoecimento dos trabalhadores é necessário refletir sobre como isso ocorre e quais são as evidências dessa problemática.

\section{Panorama das condições de trabalho e adoecimento dos trabalhadores de enfermagem}

O trabalho de enfermagem está presente 24h/dia nas instituições de saúde com internação e durante toda a jornada em outras instituições de saúde, o que torna mais intenso o impacto das condições de trabalho. Nessas instituições, há escassez de trabalhadores de enfermagem, implicando na intensificação do ritmo de trabalho e, portanto, no seu desgaste. Subsidia essa discussão o fato de que a Organização Mundial de Saúde recomenda a relação de dois enfermeiros/1.000 habitantes. Apesar do grande contingente de trabalhadores de enfermagem no País, que soma cerca de 1.500 milhão de trabalhadores ${ }^{(1)}$, essa meta ainda não foi atingida, sendo a relação de 1,42 enfermeiros/1.000 habitantes aquém da recomendada ${ }^{(2)}$. Essa escassez pode ser melhor visualizada quando se verifica que a média nacional de leitos por trabalhadores é de 21,2 enfermeiros/100 leitos, ou seja, uma média de 7 enfermeiros/100 leitos, o que não é suficiente considerando os três turnos, folgas, faltas, licenças e afastamentos desses profissionais. A escassez de trabalhadores faz com que o ritmo de trabalho seja acelerado, intensificando também o seu desgaste. O quantitativo de pessoal de enfermagem existente já é deficitário e implica em certa desqualificação da assistência, sendo imprescindível aumentar 
esse quantitativo, independentemente da carga horária semanal desses trabalhadores.

O impacto das políticas sociais nos trabalhadores de enfermagem pode ser observado quando se analisa o salário e a jornada de trabalho(3). Quanto ao salário, estudo mostra que a faixa salarial média de $50,4 \%$ dos trabalhadores de enfermagem de sete hospitais universitários(4) ${ }^{(4 i t u a-s e}$ entre $R \$ 1.001$ a $R \$ 2.000$, o que é uma remuneração muito baixa, explicando a frequência de múltiplos vínculos entre os trabalhadores de enfermagem. A média salarial por hora trabalhada para os médicos é de $\mathrm{R} \$ 23,05$; para os enfermeiros, de $\mathrm{R} \$ 11,07$; e para os técnicos e auxiliares de enfermagem, $\mathrm{R} \$ 4,15$, o que representa respectivamente $48 \%$ e $18 \%$ do salário dos médicos ${ }^{(2)}$. Os enfermeiros continuam mantendo o crescimento de emprego, porém com uma média salarial inferior à do médico e do dentista e uma jornada de trabalho semelhante à do médico. Contudo, na realidade verifica-se que os trabalhadores de enfermagem realizam uma jornada semanal maior que 44 horas semanais, uma vez que precisam cobrir ausências de outros trabalhadores, por faltas e afastamentos por acidentes e doenças. As horas que deveriam ser pagas como extras constituem um "banco de horas" nas instituições e são, geralmente, gozadas de acordo com as necessidades institucionais ou de afastamento por problemas de saúde do trabalhador, quando este teria direito a afastamento por acidente ou licença médica.

Nessas condições, os trabalhadores têm se submetido a diferentes cargas de trabalho (riscos ocupacionais) que são geradoras de processos de desgaste (adoecimento), que comprometem tanto a saúde e a vida dos trabalhadores de enfermagem como a dos pacientes e da qualidade de assistência.

\section{Exposição dos trabalhadores a cargas de trabalho e suas consequências}

Neste contexto, os trabalhadores de enfermagem estão expostos a cargas de trabalho que são determinantes dos processos de desgaste (adoecimento). Essas cargas são classificadas como: biológicas, químicas, físicas, mecânicas, fisiológicas e psíquicas ${ }^{(5)}$.

1) Cargas biológicas: acidentes com exposição aos fluídos biológicos, com potencialidade para aquisição de doenças como as hepatites, principalmente B e C; Aids etc. Têm sido notificados casos de dengue, leptospirose, febre amarela, infecções respiratórias e outras.

Estudo $^{(6)}$ mostra que por ocasião da pandemia de H1N1, houve registros de 51 suspeitos e 28 casos confirmados, com prevalência de $0,463 \%$, significativamente maior do que para a população em geral $(0,014 \%)$ e explicada pela proximidade do cuidado aos indivíduos, principalmente em portas de entrada (emergências, prontos socorros). Essa prevalência foi significativamente maior no Norte $(57,1 \%)$, diferentemente da ocorrência no País (Sudeste), lugar em que os trabalhadores de enfermagem possuem até cinco empregos, em função do baixo salário recebido, mesmo considerando a subnotificação.

2) Cargas químicas: os trabalhadores de enfermagem estão expostos a substâncias químicas na forma de medicamentos, EPI (luvas) e outras substâncias desinfetantes e esterilizantes.

Já são conhecidos os efeitos dos anestésicos, dos quimioterápicos, de alguns antibióticos e antissépticos, causadores de câncer, abortos e mal formações, assim como de alergias e de dermatites de contato (luvas de látex). Pesquisa(7) identificou 387 tipos de produtos químicos usados em uma instituição, sendo que, desses, 139 foram triados como geradores de produtos químicos perigosos: tóxicos a diferentes aparelhos e tecidos do corpo humano, carcinogênicos, teratogênicos e mutagênicos. Grande parte dessas substâncias é manuseada pelos trabalhadores de enfermagem, como o formaldeído (teratogênico, mutagênico e carcinogênico), o óxido nítrico (genotóxico), a benzina (carcinogênico), o éter (neurotóxico) e a epinefrina (teratogênico).

3) Cargas mecânicas: constituem os acidentes com agulhas e materiais perfurantes e cortantes, fraturas, torções, contusões, hematomas, muitas vezes, como resultados de violência física (bater, chutar, estapear, esfaquear, atirar objetos, empurrar, morder etc).

São relatados casos violência física em trabalhadores de enfermagem cujos agressores são os próprios pacientes e seus familiares, colegas de trabalho, (enfermagem e equipe multiprofissional). As consequências físicas da violência não são menos graves do que as psíquicas. Estudo( ${ }^{(8)}$ evidencia que, infelizmente, ocorrem desde pequenos cortes até fraturas, rompimentos de ligamentos e perda de consciência.

Apesar da visibilidade da consequência física, a subnotificação se mantém presente e frequente, devido a causas como: desconhecimento de que esse evento pode ser caracterizado como acidente de trabalho, vergonha de ser vítima, uma compreensão de que talvez isso faça parte do trabalho.

Vários estudos mostram frequências alarmantes de violência no trabalho de enfermagem ${ }^{(9,10)}$. Os trabalhadores do setor saúde são particularmente conhecidos como de risco para a violência ocupacional, com uma frequência de quase $25 \%$ do total $^{(9)}$. Estatísticas internacionais indicam que um em cada três enfermeiros é suscetível de ser submetido a abuso físico ou verbal no trabalho em comparação com um em cada quatro policiais. No Brasil, estudo transversal aponta que $100 \%$ das enfermeiras, $88,9 \%$ dos técnicos de enfermagem, $88,2 \%$ dos auxiliares de enfermagem foram vítimas de violência ocupacional ${ }^{(10)}$. 
4) Cargas fisiológicas: geradas pelo trabalho em pé, posturas inadequadas, manipulação excessiva de pesos, rodízios de turnos e trabalho noturno, são as responsáveis pela fadiga. Essa é detectada em $52 \%$ dos trabalhadores de enfermagem ${ }^{(11)}$. O trabalho de enfermagem é realizado, na maior parte da jornada, em pé, com deslocamentos frequentes, em posições inadequadas e com manipulação de pesos excessivos que representam várias toneladas, implicando na inquestionável presença de cansaço (desgate) e adoecimento.

Da exposição a essas cargas, os Distúrbios Osteomusculares Relacionados ao Trabalho (DORT) são os mais frequentes problemas de saúde, relatados por quase $100 \%$ dos trabalhadores de enfermagem. Os DORT são decorrentes do superuso das estruturas corporais para a realização do trabalho sem o devido descanso, sem as devidas pausas para recuperação. Estudo ${ }^{(12)}$ mostra que $41,5 \%$ dos trabalhadores afastaram-se por DORT, num total de 3.393 dias de afastamento.

Também o trabalho em turnos expõe os trabalhadores às cargas fisiológicas, devido às mudanças nos ritmos circadianos, advindas principalmente do trabalho noturno. A privação do sono e as mudanças dos níveis de melatonina provocam tanto gastrites, obesidade, insônia, diminuição da capacidade de julgamento e de atenção, como cânceres.

5) Cargas psíquicas: expõem os trabalhadores aos desgastes mentais, como resultado de condições inadequadas de trabalho, como relações assimétricas na equipe de enfermagem e multiprofissional, exposição à violência psíquica e outras.

A autora(12) constatou que $28,4 \%$ dos trabalhadores afastaram-se por transtornos mentais e comportamentais, representando 4.957 dias de afastamento. Outro estudo(4) refere que os transtornos mentais e comportamentais representaram $8,3 \%$, ou seja, a terceira maior causa de afastamento, que foi responsável pelo maior número de dias perdidos por problemas de saúde, devido a depressão, ansiedade, angústia, estresse e outros.

A violência psíquica é representada por assédio moral, abuso e ameaças, perseguição, assédio sexual, assédio racial e agressão psíquica.

Os episódios de violência psíquica são referidos, nacional e internacionalmente, em proporções acima de 70 a $95 \%$ da equipe $^{(13)}$, sendo a violência verbal a mais comum, expressa por sentimentos de medo, raiva, indignação, vergonha, humilhação, até a manifestação de estresse, burnout e transtorno de estresse pós-traumático, que impactam em altos custos na saúde, pelo absenteísmo e decréscimo na produtividade.

Ansiedade, estresse e burnout estão relacionados a sofrimento no trabalho pelo cuidado dos pacientes e pela violência psíquica advinda do relacionamento com os pacientes, com os pares e com a equipe multidisciplinar. Psiquicamente, a ansiedade crônica - ou esgotamento - leva a um estado de apatia e desinteresse, desânimo, uma espécie de pessimismo, insegurança e medo em relação à vida ${ }^{(14)}$, que são de difícil caracterização e expressas pelos relatos de tristeza, ansiedade, fadiga, diminuição da concentração, preocupação somática, irritabilidade e insônia.

Como causas de estresse são citadas situações que excedem o limite suportado, dentre as quais a sobrecarga de trabalho, o esforço exigido e algumas características ligadas ao exercício da profissão (gênero).

As cargas de trabalho a que os trabalhadores de enfermagem estão expostos permitem identificar esse trabalho como penoso, perigoso e insalubre.

\section{CONSEQUÊNCIAS}

Como consequência, além do adoecimento propriamente dito, as condições de trabalho e a exposição dos trabalhadores às cargas implicam em:

Diminuição da capacidade para o trabalho: estudo ${ }^{(11)}$ mostra que em trabalhadores com idade média de 39,4 anos foi constatado que $19 \%$ têm restrição de atividades; 35\% têm Índice de Capacidade para o Trabalho de moderado a baixo. Mesmo assim, os trabalhadores de enfermagem executam suas atividades com dor, desgastados, exauridos da energia vital, caracterizando o "presenteísmo" e o comprometimento com os colegas e pacientes, como mostra o estudo ${ }^{(12)}$ em que os trabalhadores só se afastam após as situações de "pico".

Absenteísmo: pesquisa ${ }^{(12)}$ mostra que $56 \%$ dos trabalhadores do quadro ausentaram-se por motivo de saúde e, destes, 91,2\% (333) eram mulheres; a idade média foi de 40,2 anos; $75 \%$ dos trabalhadores que tinham de 5 a 10 anos de trabalho; e 83,1\% tinham um único vínculo empregatício, sendo que os que tinham dois ou três vínculos representaram $16,9 \%$ dos afastamentos. Desmistifica-se assim que quem se afasta são os mais velhos, com mais tempo de casa, e que têm múltiplos vínculos.

Elevado custo dos afastamentos: as ausências por doença verificadas em estudo(12) somaram 11.941 dias, ou seja, 32 anos de trabalho perdidos em um. Se considerado o salário médio da equipe - $\mathrm{R} \$ 3.071,85$-, esses afastamentos representam 398 meses e o montante de cerca de $\mathrm{R} \$$ 1.222.596. Considerando ainda que os afastamentos até 15 dias são de responsabilidade da instituição, esses foram de 3.552 dias, o que corresponde a $\mathrm{R} \$ 363.707,04$, levando em conta apenas o salário.

Foram identificados ${ }^{(4)} 8.543$ dias perdidos por motivo de saúde, ou seja, 23 anos de trabalho perdidos em um. 
Considerando um salário médio de $\mathrm{R} \$ 2.000,00$, a perda representou, em média, $R \$ 569.553,33$.

Assim, somente considerando o salário, verificam-se os elevados custos dos afastamentos. Desmistifica-se também o alto custo que a jornada de 30 horas representaria, quando esta é comparada ao custo dos afastamentos para as instituições. Esse custo é altíssimo e poderia ser revertido para a contratação de trabalhadores de enfermagem necessários para uma jornada de 30 horas semanais.

A exposição a cargas de trabalho gera um perfil patológico que ainda é pouco conhecido pelos gerentes institucionais e órgãos da esfera pública. Também pouco se sabe a respeito de quanto esses problemas de saúde geram gastos que são pagos pela previdência social, ou seja, pela sociedade. Nesse sentido, o fato do Estado e seus cidadãos arcarem com um ônus que é das instituições que permitem o adoecimento dos seus trabalhadores é, sem dúvida, uma questão ético-política a ser discutida.

Outra consequência é a morte dos trabalhadores. Há evidências de que o câncer e o suicídio são as principais causas de morte dos enfermeiros ${ }^{(15)}$. $O$ câncer entre trabalhadores de enfermagem acomete vários sítios como: cérebro, mama, fossas nasais, sistema nervoso, pele, pulmão, fígado e outros, em decorrência da exposição a cargas químicas e trabalho noturno; o suicídio ocorre por sofrimento no trabalho, pressão, convivência com a dor e sofrimento dos outros ${ }^{(15)}$.

Ainda, outra consequência é sobre a qualidade da assistência prestada aos pacientes, onerando mais uma vez a sociedade. Pesquisa ${ }^{(16)}$ mostra a relação entre o quantitativo de pessoal e a qualidade da assistência prestada, segundo indicadores assistenciais, denotando que o quantitativo de pessoal comprometido implica no aumento das taxas de infecção, extubação acidental, mortalidade, eventos adversos e outros relativos aos cuidados prestados aos pacientes que, em última instância, impactam nos perfis de morbimortalidade da população. Além disso, as condições de trabalho implicam em um ônus individual/familiar (doença e impacto nos proventos), institucional (custo e produtividade) e social (custo social da qualidade da assistência prestada).

\section{CONSIDERAÇÕES}

Não é possível mudar a natureza do objeto de trabalho de enfermagem e das instituições de saúde que são tipicamente insalubres, assim como há limitações para instituir novas formas de organização desse trabalho. No entanto é possível controlar a insalubridade, a periculosidade e a penosidade nesse trabalho e, portanto, o desgaste e a exaustão dos trabalhadores, permitindo a recuperação da força de trabalho e o distanciamento da exposição a cargas pela diminuição da jornada de trabalho.

\section{Referências}

1. Conselho Federal de Enfermagem. Total de profissionais de enfermagem no Brasil; 2010 [Internet]. [citado em 2010 abr 28]. Disponível em: http://www. portaldaenfermagem.com.br/ estatisticas.asp

2. Ministério da Saúde (BR). Indicadores de gestão do trabalho em saúde:

material de apoio para o Programa de Qualificação e Estruturação da Gestão do Trabalho e da Educação no SUS. Brasilia: Ministério da Saúde; 2007.

3. Girardi SN, Carvalho CL. Configuraçōes do mercado de trabalho dos assalariados em saúde no Brasil. Formação. 2002;6:15-36.

4. Felli VEA, Mininel VA, Martins AC, Karino ME, Carvalho MB. Indicadores de saúde dos trabalhadores de enfermagem. Anais da XI Conferência lberoamericana de Educação em Enfermagem; 2011 set 18-24; Coimbra (Portugal); 2011. 5. Felli VEA, Tronchin DMR. La calidad de vida en el trabajo y la salud del trabajador de enfermería. In: Kurcgant P. (organizador). Gerenciamiento en enfermería. 2a ed. Rio de Janeiro: Guanabara Koogan; 2012. p. 87-105. 6. Felli V, Sarquis L, Bastos M, Baptista P, Mininel V. Respiratory diseases among nursing workers in Brazil: a report of pandemic influenza. In: Anais 30th International Congress on Occupational Health; 2012 mar 18-23; Cancun (México); 2012.

7. Costa TF. Gerenciamento de resíduos químicos perigosos manuseados pela enfermagem de um hospital universitário [tese]. São Paulo: Escola de Enfermagem da USP; 2009

8. Bastos M, Felli VEA. Danos da violência ocupacional: revisão integrativa da literatura. Relatório de pesquisa. São Paulo: [s.n.]; 2012.
9. Di Martino V. Workplace violence in the health sector. Report. Geneve: [s.n.]; 2002.

10. Cezar ES, Marziale MHP. Problemas de violência ocupacional em um serviço de urgência hospitalar da Cidade de Londrina, Paraná, Brasil. Cad Saúde Pública. 2006;22(1):217-21.

11. Silva FJ, Felli VEA, Sarquis LMM, Costa TF, Tito RS. Avaliação da capacidade para o trabalho entre trabalhadores de enfermagem. In: Anais da XI

Conferência Iberoamericana de Educação em Enfermagem; 2011 set 18-24; Coimbra (Portugal); 2011.

12. Sancinetti TR. Absenteísmo por doença na equipe de enfermagem: taxa diagnóstico médico e perfil profissional [tese]. São Paulo: Escola de Enfermagem da USP; 2009.

13. Hahn S, Müller M, Needham I, Dassen T, Kok G, Halfens RJG. Factors associated with patient and visitor violence experienced by nurses in general hospitals in Switzerland: a cross-sectional survey. J Clin Nurs. 2010;19 (23-24):3525-46.

14. Ballone GJ, Ortoloni IV. Comportamento violento [Internet]. [citado em 2012 abr 14]. Disponível em: http://virtualpsy.locaweb.com.br/index. php?sec $=9$ \&art $=34$

15. Karino ME. As causas de morte dos enfermeiros: uma revisão sistemática [tese]. São Paulo: Escola de Enfermagem da USP; 2012.

16. Penoyer DA. Nurse staffing and patient outcomes in critical care: a concise review. Crit Care Med. 2010; 38 (7):504-9. 